\title{
Ultrasound assessment of the myofascial trigger point. A systematic review
}

\author{
López San Miguel G. ${ }^{1}$ Barbe Mendibil I. ${ }^{1}$ Torres Chica B. ${ }^{1}$ Ríos Diaz J. ${ }^{1}$ \\ 1 Physiotherapy Center Eduardo Álvarez, Asturias, Spain
}

Rev Fisioter Invasiva 2019;2:68.

\begin{abstract}
Introduction Myofascial pain syndrome is an important and prevalent public health problem. The lack of consensus on the diagnostic criteria, together with the scarce reliability of the manual detection of the manual detection of myofascial trigger points (MTrPs) point to the need to develop objective methods to enable confirmation of the presence of MTrPs. Ultrasound is an accessible method which enables the assessment of tissue properties in real time, helping to characterize the MTrP, understand its physiopathology and define its diagnosis.

Aims To identify observational studies researching the use of ultrasound in the assessment of MTrPs. Also, to learn about and compile the advances in the study of the characteristics of MTrPs and their sonographic diagnosis.

Material and Methods A systematic review was performed by two independent reviewers, searching biomedical databases using terms related with "ultrasound" and "trigger points". Observational studies were selected evaluating the characteristics of MTrPs. Subsequently, an analysis of the diagnostic quality of studies was performed using the QAREL scale and a study of the methodological quality took place based on the Downs and Black scale. Furthermore, an assessment of the reproducibility of the acquirement of images was performed, via the analysis of the description of the ultrasound method. The risk of bias was evaluated according to the Cochrane guidelines.

Results 18 studies based on B Mode methods, elastography and Doppler, were included in the review. The anatomic regions which were most explored were the cervical area and the upper limb, evaluated in 14 of the 18 papers. The most common muscle was the upper trapezius (61\%). Two articles were located corresponding to the lumbar region and one article concerned the lower limb. The analysis of the sonographic method showed a low level, 5 of the 9 items did not overcome $17 \%$ of fulfillment, in 3 papers the frequency was below $6 \%$. The QAREL scale also displayed low levels, only 3 items out of 11 . Inter-evaluator blinding, correct testing and statistical methods were fulfilled in over $50 \%$. The mean score obtained by the studies in the Downs and Black scale was 5.4 points out of 10 , ranging between 2 and 7 points. The risk of bias according to the Cochrane guidelines was mid- to high.

Conclusion Important steps have been taken in the study of the sonograhic character-

\section{Keywords}

- ultrasonography

- trigger points

- myofascial pain syndrome istics of the MTrP, however, we are still far from standardizing the use of the same as a diagnostic method. The poor results in the quality analysis of the present study suggest caution in the interpretation of the present findings. Future research is necessary, including different anatomic regions, analytic methods, better defined exploration protocols and more robust reliability studies for the different methods available.
\end{abstract}

DOI https://doi.org/ $10.1055 / \mathrm{s}-0039-3401889$. ISSN 2386-4591.
Copyright $\odot 2019$ by Thieme Revinter

Publicações Ltda, Rio de Janeiro, Brazil
License terms

(®) (1) $\Theta \circledast$ 